\title{
ARTICLES \\ FURTHER RESULTS ON HESSELAGER'S RECURSIVE PROCEDURE FOR CALCULATION OF SOME COMPOUND DISTRIBUTIONS *
}

\author{
By Shaun Wang \\ University of Waterloo, Canada \\ And Monica Sobrero \\ Università “'La Sapienza", Italy
}

\section{ABSTRACT}

The recursive algorithm of HESSELAGER (1994) is extended to a more general class of counting distributions, which includes SUNDT's (1992) class as well as all the mixed Poisson distributions discussed by WiLLMOT (1993).

\section{KEYWORDS}

Compound distributions; recursions; mixed Poissons; Sichel; Beta; Generalized Pareto; Inverse Gamma.

\section{INTRODUCTION}

In the collective risk model, compound distributions are used extensively in modeling the total claim for an insurance portfolio:

$$
S=\sum_{i=1}^{N} X_{i},
$$

where the claim sizes $X_{i}$ 's are independent and identically distributed and independent of the claim frequency $N$.

If the claim frequency $N$ has a probability function (p.f.) $\left\{p_{0}, p_{1}, \ldots\right\}$, and the claims sizes $\left\{X_{1}, X_{2}, \ldots\right\}$ have a common p.f.:

$$
f_{x}=\operatorname{Pr}\{X=x\}, \quad x=0,1, \ldots,
$$

then the total claim $S$ has a compound distribution with a p.f.

$$
g_{x}=\sum_{n=0}^{\infty} p_{n} f_{x}^{* n} .
$$

Since PANJER (1981), many resursive algorithms have been derived for a broad class of claim frequency distribtions (see Willmot and PANJER, 1987; SUNDT, 1992; Willmot, 1993; and others).

* The authors are grateful to the editor and the referees for their helpful comments. 
HESSELAGER (1994) recently considered a class of claim frequency distributions satisfying :

$$
p_{n}=\frac{\sum_{i=0}^{k} a_{i} n^{i}}{\sum_{i=0}^{k} b_{i} n^{i}} p_{n-1}, \quad n=1,2, \ldots
$$

for some positive integer $k$, and derived recursions for the related compound distributions. For some counting distributions such as Generalized Waring, Hypergeometric and Polya-Eggenberger, Hesselager's method is more efficient than the ones provided by WILLMOT and PANJER (1987). However, Hesselager's class (3) does not include many other counting distributions such as Sichel, Poisson-Beta, Poisson Generalized Pareto, Poisson Inverse Gamma, Poisson Transformed Gamma and Poisson Transformed Beta (see Willmot, 1993).

In this paper, we extend Hesselager's recursive scheme to a broader family of counting distributions, which includes al the counting distributions which satisfy a finite order homogeneous recursion with polynomial coefficients. All the mixed Poisson distributions in WILlmot (1993) are of this type (where the nonhomogeneous terms can be eliminated).

\section{RECURSIONS FOR THE EXTENDED CLASS OF COUNTING DISTRIBUTIONS}

Assume that the claim frequency $N$ has a p.f. $\left\{p_{0}, p_{1} \ldots\right\}$ satisfying:

$$
\left(\sum_{i=0}^{k} b_{i} n^{i}\right) p_{n}=\sum_{j=1}^{s}\left\{\sum_{i=0}^{k} a_{j, i}(n-j)^{i}\right\} p_{n-j}, \quad n=c, c+1, \ldots
$$

where $c$ is a positive integer and $p_{n}=0$ for $n<0$.

Hesselager (1994) introduced the following auxiliary functions

$$
g_{i, x}=\sum_{n=0}^{\infty} n^{i} p_{n} f_{x}^{* n}, \quad x=0,1, \ldots ; \quad(i=0,1, \ldots)
$$

(with $0^{0}=1$ ) and defined the vector

$$
\vec{g}_{x}=\left(g_{0, x}, \ldots, g_{k, x}\right)^{\prime} .
$$

Note that $g_{0, x}$ is the p.f. for the total claim distribution of $S$ in (2).

Before we generalize Hesselager's result, we first introduce another auxiliary function :

$$
\Omega(x)=\sum_{n=1}^{c-1} \sum_{i=0}^{k} b_{i} n^{i} p_{n} f_{x}^{*^{n}}-\sum_{j=1}^{s} \sum_{n=j}^{c-1} \sum_{i=0}^{k} a_{j, i}(n-j)^{i} p_{n-j} f_{i}^{* n},
$$

where $\sum_{n=j}^{c-1}$ is zero if $j>c-1$. By letting $a_{0, i}=-b_{i}$ the expression for $\Omega(x)$ may be written as

$$
\Omega(x)=-\sum_{j=0}^{s} \sum_{n=j}^{c-1} \sum_{i=0}^{k} a_{j, i}(n-j)^{i} p_{n-j} f_{x}^{* n} .
$$


Note that $\Omega(x)$ does not depend upon the values of $\vec{g}_{x}$. In the special case of $k=s=c=2$, we have

$$
\Omega(x)=p_{1} f_{x} \sum_{i=0}^{2} b_{i}-p_{0} f_{x} a_{1,0} .
$$

As in Hesselager (1994), let $m$ be the smallest integer for which $f_{m}>0$.

Theorem 1. For the claim frequencies in (4), the compound distribution $g_{x}=g_{0, x}$ can be evaluated by the following recursive method:

$$
\vec{g}_{x}=T_{x}^{-1} \vec{t}_{x}, \quad x \geq m \vee 1,
$$

where

$$
T_{x}=\left(\begin{array}{ccccc}
1 & -m / x & 0 & \ldots & 0 \\
0 & 1 & -m / x & \ldots & 0 \\
\vdots & \vdots & \vdots & \vdots & \vdots \\
\left(b_{0}-\sum_{j=1}^{s} a_{j, 0} f_{0}^{j}\right) & \left(b_{1}-\sum_{j=1}^{s} a_{j, 1} f_{0}^{j}\right) & \ldots & \ldots & \left(b_{k}-\sum_{j=1}^{s} a_{j, k} f_{0}^{j}\right)
\end{array}\right),
$$

and $\vec{t}_{x}=\left(t_{0, x}, \ldots, t_{k, x}\right)^{\prime}$ is given by

$$
\begin{gathered}
t_{i, x}=\frac{1}{f_{m}} \sum_{y=1}^{x} f_{m+y}\left\{\frac{m+y}{x} g_{i+1, x-y}+\frac{y-x}{x} g_{i, x-y}\right\}, \quad i<k ; \\
t_{k, x}=\sum_{y=1}^{x} \sum_{i=0}^{k} \sum_{j=1}^{s} a_{j, i} f_{y}^{* j} g_{i, x-y}+\Omega(x),
\end{gathered}
$$

with starting values

$$
\begin{array}{rlrl}
g_{i, 0}=\sum_{n=0}^{\infty} n^{i} p_{n} f_{0}^{n}, & i=0, \ldots, k ; \\
g_{i, x}=0, & i=0, \ldots, k ; & x=1, \ldots, m-1 .
\end{array}
$$

Proof: Let $F(z)=\sum_{x=0}^{\infty} f_{x} z^{x}$ be the probability generating function for the claim size distribution.

From the identity $\frac{d}{d z}\left[F(z)^{n}\right]=n F(z)^{n-1} F^{\prime}(z)$, we have

$$
0=\sum_{y=0}^{x}\left[(n+1) \frac{y}{x}-1\right] f_{y} f_{x-y}^{* n} .
$$

Multiplying (12) by $p_{n} n^{i}$ and summing over $n \geq 0$ yields

$$
0=\sum_{y=0}^{x} f_{y}\left\{\frac{y}{x} g_{i+1, x-y}+\left(\frac{y}{x}-1\right) g_{i, x-y}\right\} \text {. }
$$


By omitting the zero terms corresponding to $y=0, \ldots, m-1$ and taking out the terms involving $\vec{g}_{x}$, we get for $i<k$,

$$
g_{i, x}-\frac{m}{x} g_{i+1, x}=t_{i, x},
$$

where $t_{i, x}$ is given in (10).

Note that $f_{x}^{*^{n}}=\sum_{y=0}^{x} f_{y}^{* j} f_{x}^{* *_{y}^{(n-j)}}$ for $j=1, \ldots, s$.

Multiplying the left-hand side of (4) by $f_{x}^{* n}$, multiplying the $j$-th term of the right-hand side of (4) by $\Sigma_{y=0}^{x} f_{y}^{* j} f_{x-y}^{*(n-j)}$, and summing over $n \geq c$, we obtain the relation (for $x \geq 1$ ):

$$
\begin{aligned}
\sum_{i=0}^{k} b_{i} g_{i, x} & -\sum_{n=1}^{c-1} \sum_{i=0}^{k} b_{i} n^{i} p_{n} f_{x}^{* n}=\sum_{y=0}^{x} \sum_{i=0}^{k} \sum_{j=1}^{s} a_{j, i} f_{y}^{* j} g_{i, x-y} \\
& -\sum_{j=1}^{s} \sum_{n=j}^{c-1} \sum_{i=0}^{k} a_{j, i}(n-j)^{i} p_{n-j} f_{x}^{* n} .
\end{aligned}
$$

By collecting the leading terms involving $g_{i, x}$, we get

$$
\begin{aligned}
\sum_{i=0}^{k}\left(b_{i}\right. & \left.-\sum_{j=1}^{s} a_{j, i} f_{0}^{j}\right) g_{i, x} \\
& =\sum_{y=1}^{x} \sum_{i=0}^{k} \sum_{j=1}^{s} a_{j, i} f_{y}^{* j} g_{i, x-y}+\Omega(x), \quad x \geq 1,
\end{aligned}
$$

where $\Omega(x)$ is as defined in (6).

From equations (14) and (15), we obtain the linear equations (8).

Remark 1: As a special case, for $m=0$ (i.e. $f_{0}>0$ ), we have the following recursions :

$$
\begin{aligned}
& g_{i, x}=\frac{1}{f_{0}} \sum_{y=1}^{x} f_{y}\left\{\frac{y}{x} g_{i+1, x-y}+\frac{y-x}{x} g_{i, x-y}\right\}, \quad i<k \\
& g_{k, x}=\frac{1}{b_{k}-\sum_{j=1}^{s} a_{j, k} f_{0}^{j}} \times \\
&\left\{\sum_{y=1}^{x} \sum_{i=0}^{k} \sum_{j=1}^{s} a_{j, i} f_{y}^{* j} g_{i, x-y}+\sum_{i=0}^{k-1}\left(\sum_{j=1}^{s} a_{j, i} f_{0}^{j}-b_{i}\right) g_{i, x}+\Omega(x)\right\} .
\end{aligned}
$$

Remark 2: HeSSLAGER's (1994) recursive formula can be recovered from (8) as a particular case of $s=c=1$.

3. eXAMPLes OF MiXed PoISSON Distributions

Mixed Poisson distributions are natural candidates for modeling the claim frequency for heterogenous risk portfolios. Willmot (1993) considered various mixed Poisson 
distributions and derived recursions for their probability functions. Some of the mixed Poisson distributions belong to the Pearson system (see ORD, 1972, p. 8) and WILLMOT (1993) derived recursions for their compound distributions. However, many mixed Poisson distributions, which are not in the Pearson system, belong to the generalized class (4). So our generalized recursive procedure can be used in the calculation of the compound distributions for these mixed Poisson frequencies.

Example 1: The Sichel distribution is obtained by mixing the Poisson mean over the Generalized Inverse Gaussian. WILLMOT (1993) derives a recursion for this mixed Poisson p.f. (for $n=2,3, \ldots$ )

$$
(1+2 \beta) n(n-1) p_{n}=2 \beta(n-1)(n+\lambda-1) p_{n-1}+\mu^{2} p_{n-2},
$$

which corresponds to (4) with $k=s=c=2$ and

$$
\begin{array}{lll}
b_{0}=0, & b_{1}=-(1+2 \beta), & b_{2}=1+2 \beta ; \\
a_{1,0}=0, & a_{1,1}=2 \beta \lambda, & a_{1,2}=2 \beta ; \\
a_{2,0}=\mu^{2}, & a_{2,1}=0, & a_{2,2}=0 .
\end{array}
$$

Example 2: The Poisson Beta is obtained by mixing the Poisson mean over the Beta distribution. WILLMOT (1993) derives a recursion for this mixed Poisson p.f. (for $n=2,3, \ldots$ )

$$
n(n-1) p_{n}=(n-1)(n-2+\mu+\alpha+\beta) p_{n-1}-\mu(n-2+\alpha) p_{n-2},
$$

which corresponds to (4) with $k=s=c=2$ and

$$
\begin{array}{lll}
b_{0}=0, & b_{1}=-1, & b_{2}=1 ; \\
a_{1,0}=0, & a_{1,1}=\mu+\alpha+\beta-1, & a_{1,2}=1 ; \\
a_{2,0}=-\mu \alpha, & a_{2,1}=-\mu, & a_{2,2}=0 .
\end{array}
$$

Example 3: The Poisson Generalized Pareto is obtained by mixing the Poisson mean over the Generalized Pareto. WILlmot (1993) derives a recursion for this mixed Poisson p.f. (for $n=2,3 \ldots$ )

$$
n(n-1) p_{n}=(n-1)(n-1-\alpha-\mu) p_{n-1}+\mu(n-2+\beta) p_{n-2},
$$

which corresponds to (4) with $k=s=c=2$ and

$$
\begin{array}{lll}
b_{0}=0, & b_{1}=-1, & b_{2}=1 ; \\
a_{1,0}=0, & a_{1,1}=-(\alpha+\mu), & a_{1,2}=1 ; \\
a_{2,0}=\mu \beta, & a_{2,1}=\mu, & a_{2,2}=0 .
\end{array}
$$

Example 4: The Poisson Inverse Gamma is obtained by mixing the Poisson mean over the Inverse Gamma. WiLlmot (1993) derives a recursion for this mixed Poisson p.f. (for $n=2,3, \ldots$ )

$$
n(n-1) p_{n}=(n-1)(n-1-\alpha) p_{n-1}+\mu p_{n-2},
$$


which corresponds to (4) with $k=s=c=2$ and

$$
\begin{array}{lll}
b_{0}=0, & b_{1}=-1, & b_{2}=1 ; \\
a_{1,0}=0, & a_{1,1}=-\alpha, & a_{1,2}=1 ; \\
a_{2,0}=\mu, & a_{2,1}=0, & a_{2,2}=0 .
\end{array}
$$

Remark: In all the above examples $\Omega(x)$ vanishes, which simplifies equation (8). However, $T_{x}$ becomes a singular matrix at $x=m$, so the recursive evaluation by (8) should start with initial values $\left\{\vec{g}_{0}, \ldots, \vec{g}_{m}\right\}$. Other mixed Poisson distributions in the generalized class (4) are Poisson Transformed Gamma and Poisson Transformed Beta (see Willmot, 1993).

It is noted that SunDT's (1992) class is a particular case of (4) when $k=1$. However, when $k=1$, Sundt's recursion involves only $g_{0, x}$, while recursion (8) requires both $g_{0, x}$ and $g_{1, x}$. In the same way, for mixed Poisson distributions in the Pearson system, Willmot's recursive method (see WILLMOT, 1993) is simpler than recursion (8) in evaluating their compound distributions. On the other hand, when $k \geq 2$, as in the earlier examples of this section where $k=s=c=2$, the recursion (8) is more efficient than the ones given in WILLMOT and PANJER (1987).

The numerical aspects such as stability concerns of the recursion (8) need further study.

\section{REFERENCES}

Hesselager, O. (1994) A recursive procedure for calculation of some compound distributions. ASTIN Bulletin, to appear.

ORD, J. K. (1972) Families Of Frequency Distributions. Griffin, London.

PANJER, H.H. (1981) Recursive evaluation of a family of compund distributions. ASTIN Bulletin 12, $22-26$.

SUNDT, B. (1992) On some extensions of Panjer's class of counting distributions. ASTIN Bulletin 22, $61-80$.

Willmot, G. E. (1993) On recursive evaluation of mixed Poisson probabilities and related quantities. Scandinavian Actuarial Journal, to appear.

Willmot, G.E. and PANJER, H.H. (1987) Difference equation approaches in evaluation of compound distributions. Insurance: Mathematics and Economics 6, 43-56.

The mailing addresses of the authors are:

SHAUn Wang

Dept. of Statistics \& Actuarial Science

University of Waterloo

Waterloo, Ontario, Canada N2L $3 G 1$

AND MONICA Sobrero

Dipartimento di Scienze Attuariali e Matematica

Facoltà di Economia e Commercio

Università "La Sapienza", Roma, Italia 